action. An essentially mechanical cause has to be sought for locally, and thrombosis, due either to coagulation or laceration, seems to account for the matter satisfactorily.

I have to thank Mr. A. Shillitoe, registrar to the London Lock Hospital, for the careful notes of the above case. He has also looked up and arranged the twelve cases given in the accompanying table; these are interesting especially from the point of view of treatment. These cases, with that already described, give thirteen examples of prolonged priapism, not the result of any cerebro-spinal lesion. Three were complicated with splenic trouble. In only four is the condition stated to have followed on intercourse. In three the final result was impotence. In Case 3 the organ is said to have afterwards performed its double functions satisfactorily. In the other cases the impairment or otherwise of function is not remarked upon. In the Nen York Medicu? Jontrnal for $1888 \mathrm{Mr}$. George Peabody said he had collected thirty-two cases of prolonged priapism, six of which were complicated with enlarged spleen and its results. Among the six are a case under the care of Dr. Klemme (Scbmidt's "Jahrbücher," 1866) ; one under the care of Dr. Louquet (Allgemeine Medicinische Central-Zeitung, 1876); and one under the care of Dr. Matthias (Berliner Klinische Wocleenschrift, 1879).

Hertford-street, Mayfair,.W.

\section{ACCIDENTS ATTENDING VACCINATION.}

BY P. MURRAY BRAIDWOOD, M.D. EDIN.,

COXSULTING MEDICAL OFFICHR TO THE WIRRAL FOSPITAI, FOR SICK CHILDREN.

From a copy of the "Inquiry into the Causes and Effects of the Variolæ Vaccinæ" (third edition, 1801, p. 5) I learn from the discoverer of vaccination that when milkmen and milkmaids get infected by vaccinia their systems become affected, "the pulse is quickened; shiverings, succeeded by heat, general lassitude, and pains about the loins and limbs, with romiting, come on. The head is painful, and the patient is now and then even affected with delirium." This graphic description would apply to an attack by any of the causes which induce constitutional disturbance. It is therefore of no wonder that vaccinia, even in the mild form of arm to arm vaccination, is occasionally attended and followed by accidents. Jenner always maintained febrile reaction to be "the only perfect proof of constitutional affection and absolutely essential to the perfection of the prophylaxis." Without it he considered a vaccination to have been "merely local and quite or nearly without value." Again, in animal vaccination, where the vaccine has been continuously transmitted through heifers without having ever been in contact with human blood, the vaccinia pursues a definite course, papulation appears at the end of the third or beginning of the fourth day, areolation commences at the latter end of the ninth or beginning of the tenth day, the formation of crust or scab being accomplished on the sixteenth or seventeenth day, and failing off spontaneously about the $t$ wenty.fifth day. In children vaccinated with humanised lymph the stages of vaccinia are of much shorter duration. The crust resulting from animal vaccinia approaches very nearly the size of the vesicle, is of a rich brown or dark mahogany colour, and when employed for raccinating children shows great reluctance to affect the buman system. In animal vaccination there is noticeable very decided febrile reaction coincident with the rise, development, and decline of the areola, and the disease results in an indelible cicatrix or scar of a peculiar and well-defined type. On the other hand, vaccinia induced by humanised lymph runs a rapid course, its vesicles are easily ruptured, the crust is of much smaller size than the vesicles, falls off early, and the cicatrix forms a comparatively imperfect mark. It is well, further, to bear in mind the bovine origin of vaccinia, its relationship to diseases in the lower animals, its epidemic character when spontaneously generated in the bovine economy, but not when introduced there by inoculation; its analogy to, but not fraternity with, human epidemic affections, and its inoculability both into the lower animals and into man.

1 The Morphology of Vaccine Lymph, by P. M. Braidwood, I.D. Edin., in the British and Foreign Medico-Chirurgical Review, January, 1873.
It is no uncommon occurrence for a child to be exposed to measles, varicella, \&c., and at the same time to be vaccinated, so that he is under the influence of two contagia, and the result depends on which of the two contagia takes the lead. "If," says Dr. Seaton, "vaccination has been successfully performed during the incubation of measles, but the vesicles have not reached their mature or areolar stage by the time that the disease manifests itself, it will very frequently, if not usually, be found that they will not go through that stage until such time as the measles have subsided." Jenner gives a very interesting account of two sisters in whom scarlet fever manifested itself on the eighth day after vaccination. In one the vaccine vesicle remained at its maturity, but " there was a total suspension of areola till the scarlatina had retired from the constitution"; as soon as this occurred the areola advanced in the usual way. In the sister the symptoms indicative of scarlet fever came on severely for about twelve hours; then a rash showed itself upon the face and part of the neck, but in two or three hours suddenly disappeared, and the patient was free from every complaint; it was then observed that the vesicle had the usual areola, and this continued till the fourth day, when it subsiced, and scarlet fever again appeared and went through its course with the common symptoms. Vaccination has been repeatedly seen running its course along with scarlet fever, or measles, or chickenpox. In the case of concurrent variola and vaccinia, if both diseases obtained equal mastery of the system, each pursued its own course as far as the local phenomena were concerned, though even then the constitutional effects of the small-pox were often somewhat modified. If, however, the small-pox had got a distinct mastery of the system before the cow-pox it was unaffected by the latter; and the cow-pox did not exhibit its full local phenomena when the system was under the influence of the small-pox contagion. If, however, vaccinia precedes variola it modifies the latter, or altogether prevents its development. Comparative pathology often exemplifies this. Taccine lymph produces vaccinia, and no disease but vaccinia. These remarks, preliminary to the subject matter proper of this paper, seem to me to be called for by the hazy knowledge of vaccination I bave met with among young practitioners and from the manner of teaching it I have experienced.

As regards, now, some accidents which may attend this great earthly blessing (vaccination). In the first place, the vaccination may not "take"; that means that the infective matter (vaccine) applied to the scratched surface of the skin does not affect the system and does not produce the desired vesicles. This accident may be due to the lymph being rubbed off before it has been ahsorbed, and can be avoided with a little care. The lymph should be thoroughly dried on the surface of the skin before the vaccinated spots are covered. Or the vaccine may not be "active" owing to its being not quite fresh, and having been stored too long. It sbould be used as soon as possible after removal from the vaccinifer. Secondly, in the case of vaccination of adults it may not succeed because they bad been successfully vaccinated in infancy, and again about the age of fifteen or later. Thirdly, it seems hard cccasionally to account for vaccination not succeeding till, a few days later, the child or the adult develops some other form of fever. For example, after a child has been carefully and properly vaccinated the vesicles do not appear, but he shows the morbillar or scarlatinal rash, or develops pneumonia. Either of these diseases had first taken hold of his system and prevented the vaccine lymph from having its effect. It is most important to learn from those who bring children to be vaccinated that they are perfectly healthy and well, and have not recently been exposed to infection. Adults, too, should bear in mind that they ought to be quite well before being vaccinated.

I have already mentioned that vaccinia affects the body as other constitutional diseases do ; bence it is needful to avoid colds or other accidents which may interfere with its running the natural course. Sometimes as the vaccinia abates the affected arm becomes very red, swollen, and painful, even down to the elbow, while there is sympathetic axillary lymphangitis. At its commencement this distressing condition can be relieved by painting the surface of the arm once or twice lightly with spirit of terebinth, avoiding, of course, the seat of vaccination. A saline aperient is also useful when the constitutional symptoms are severe. The amount and extent of the inflammation attending vaccination varies according to the constitution and to the state of health of the person or child. From the third or fourth day 
after vaccination, when the vesicles begin to form, the vaccinated spots should be protected from abrasion by stitching some cotton-wool on the inside of the sleeve. Vaccination shields are in my experience very hurtful. As vaccinia finishes its course troubles often arise through the vaccinated spots not healing rapidly. Various circumstances account for this, the most important being that the crust is rubbed off during sleep. If, then, the vaccinated spots are not protected, not only are they liable to receive surrounding impurities, but may lead to auto-inoculation. In the out-patient department of hospitals such cases are frequently met with, and the infant's mother wrongly ascribes it to the vaccine. Such impetiginous eruptions are really due to ill-health and uncleanliness and may appear on any part of the body. A form of eczema often attacks infants' heads and likewise is ascribed to vaccinia without reason. After the vaccine has dried on the scarified spots the infant can have his usual bath, but on no account should moist applications be used over the vaccinated spots. It should be remembered, too, that the irritation of teething often accompanies vaccinia.

The principal causes of bad and unprotective vaccination are, however, ascribable to the operator-the use of lymph taken at too late a period in the course of the disease, vaccinating with lymph removed from vesicles themselves badly developed or imperfect in character, the employment of lymph too long preserved, the use of a rusty or dirty lancet, and the manner of operating so that no blood is drawn and the lymph does not reach the cutis vera. It should be borne in mind that lymph from an exhausted vesicle is imperfect in power. It is improper, too, to vaccinate with lymph removed from a vesicle after the areola has been fairly formed, and it is decidedly injurious to vaccinate with lymph which is undergoing suppurative deterioration. An occasional accident attending vaccination is erysipelas.

In order to vaccinate always successfully great attention should be paid to details, and it requires a very decided degree of technical skill, patience, and experience. This is the more important when heifer vaccine is coming into general use. Its action is stronger than that of humanised vaccine.

Trevenson, Aylesbury.

\section{NOTES ON THE TREATMENT OF SEA-SICKNESS.}

BY M. CHARTERIS, M.D. EDIN.,

PROFESSOR OF MATERTA MEDICA AND THERAPEUTYCS AT THF UNIVERSITY OF GLASGOW.

IN my winter lectures it has been my practice to speak of the climatic treatment of disease, and notably of the magic effect produced by sea voyages. I had also to point out that in many cases the patient's liability to sea-sickness prevented the full use of this the best of nature's tonics, and in order to show my students how this disadvantage may be removed I have drawn their attention to the preparation called chlorobrom, which, as I know from personal experience, in many cases cures, and in all cases alleviates, sea-sickness. From time to time reports on its efficacy are sent to me by old students who have had experience at sea, and I have now some fresh cases to communicate.

Six ship surgeons previously prone to sea-sickness, who during the last six months have, by following my directions, sailed to Calcutta and back without suffering the least inconvenience, report as follows.

Examples of propluflactic treatment. - (a) Dr. Dunlop writes on Jan. 13th, 1897 :- "I am an indifferent sailor, and the prospect of a voyage to India in the capacity of a surgeon was marred by the thought that $I$ would suffer from mal de mer. By following the directions given me as to a cholagogue, dietary, and dosage of chlorobrom, I suffered no ill effects from the voyage." (b) Dr. McLure, on Jan. 23rd, 1897, reported as follows:- "Ordinarily a poor sailor, by acting on your directions I was enabled to reach Calcutta without the slightest feeling of nausea, and although on the homeward voyage we had a very rough passage across the Bay of Biscay, I was equally free from any feeling akin to nausea or sickness." (c) Dr. McNaught, on Sept. 25th, 1896, wrote inter alia: "In my own case I had an opportunity of trying chlorobrom as a prophylactic remedy, and $I$ feel sure it was the means on several occasions of warding off unpleasant attacks of sea-sickness." The others testify in a similar manner. (d) Dr. Allan gives the following account of a case of prophylactic treatment: "A lady who had always suffered from sickness when the surface of the waters was troubled, was treated as follows. Before sailing she took, according to my advice, a smart calomel purge ; on retiring to her cabin in the evening she had one drachm of chlorobrom ; next morning a light breakfast and then another drachm of chlorobrom. At noon-the steamer beginning to roll-she had three drachms, and I asked her to lie down for an hour or two: she did this. She at no time felt sick, and in the course of the afternoon she expressed her astonishment and gratitude at such unaccustomed immunity."

Examples of curative treatment. CASE 1.-A man was very sick one hour after putting to sea. He had just partaken of breakfast. After the stomach was cleared he had a glass of soda water. Five minutes afterwards he took twenty. five minims of spiritus chloroformi. The stomach being now soothed, he had two drachms of chlorobrom and one drachm every half hour for three doses. About noon he fell asleep and awoke at 4 P.M. He had one drachm of chlorobrom and also three grains of calomel. He felt thoroughly well, and at 6.30 P.M. he sat down to dinner and enjoyed a hearty meal. The same course was pursued later when he sickened in the monsoon, and a similar happy result was obtained.

CASE 2.-A man, who had been previously free from any symptom of sickness, complained of depression and what 1 called mental pain and nausea. After taking a dose of calomel he lay down, and half-an-hour afterwards he was given half an ounce of chlorobrom. Towards night he felt better. At 8 P.M. and at 9 P.M. he took one drachm, and at 10 P.I. two drachms of chlorobrom. Next morning he was up early and, as be expressed it, " as lively as a cricket." For the next three nights he had one drachm of chlorobrom. At no time had he any return of the symptoms.

These cases are almost picked at random and are illustrations of the efficacy of chlorobrom. I have no hesitation in saying that, if administered in a sensible and thorough manner, chlorobrom will effectively meet each and every symptom of the dreaded sickness. And I am glad to give. my testimony of the success of a treatment which I learned. within the walls of my University.

Glasgow.

\section{Clinital atotes:}

MEDICAL, SURGICAL, OBSTETRICAL, AND THERAPEUTICAL.

\section{A CASE OF FOREIGN BODY IN A BRONCHUS.}

By T. LACNCELOT ARCHER, M.R.C.S. ENG.

IN November, 1895, I was sent for to see a boy, age nine years, who had been ailing for a few days, gradually? getting worse. The temperature was $103^{\circ} \mathrm{F}$, there was a. rapid, full pulse, the expression was anxious, and he had a short spasmodic cough without expectoration. The lower lobe of the left lung was congested, but all the remainder of the lungs was clear. I saw the child daily for more than a week, during which time his temperature varied but little, the congestion did not clear up, and the cough was, if anything, worse, added to which there were violent retching and some diarrhœa. He also complained of great pain about the level of the ensiform cartilage on the left side. Eleven days after my first visit I received an urgent message saying that the child was choking. When I arrived he was pale and exhausted, but quiet. His mother said that be had had a violent fit of coughing, lasting for twenty minutes, followed by some blood-stained expectoration. On examining the sputa I found a piece of a nut of the size of a large bean, surrounded by blood-stained purulent matter and mucus of very offensive smell. The child passed a quiet night, coughing only three or four times, but expectorating purulent matter, and in the morning he was decidedly better : from that time he steadily improved to complete recovery. 